\title{
Detection of efflux pump in multidrug resistant acinetobacter Species using Naphthyl-Methyl-Piperazine
}

\author{
Sandeep T. ${ }^{1, *}$, Indumathi V. A. ${ }^{2}$, Prasanthi S. ${ }^{3}$ \\ ${ }^{1}$ Assistant Professor, ${ }^{2}$ Professor, ${ }^{3}$ Senior manager-Biostatistics, ${ }^{1,2}$ Dept. of Microbiology, M.S. Ramaiah Medical College, \\ Bengaluru, Karnataka, ${ }^{3}$ Covance India Pvt. Ltd., Bengaluru, Karnataka, India
}

*Corresponding Author:

Email: dr.sandeept@yahoo.co.in

\begin{abstract}
Acinetobacter, Gram negative bacterium, has proven to be recently important to research due to multidrug resistance. Multidrug resistance is directed against antibiotics like Aminoglycosides, Carbapenems, Cephalosporins, Quinolones etc. The presence of efflux pump, primarily the Resistance-Nodulation-Cell Division (RND) type, is an active transport mechanism which allows it to efficiently reduce antibiotic levels within the cell. 1-(1-naphtylmethyl)-piperazine (NMP) is an efflux pump inhibitor (EPI) which mechanically inhibits the efflux pump in bacteria resulting in increased concentration of antibiotic intracellularly, thereby killing the cell.

Objectives: To detect the efflux pumps phenotypically using NMP and to establish the cut-off zone diameter to detect efflux pump in Acinetobacter species.

Materials and Methods: Acinetobacter species were isolated from patient samples using conventional techniques of inoculation, identification and antibiotic susceptibility testing. The efflux pump was detected phenotypically by disc diffusion method on Mueller Hinton agar with NMP and chloramphenicol, tetracycline discs as substrates. The zone diameters were recorded \& values compared using t-test. Tipping analysis was done to determine the mean zone difference to identify the presence of efflux pump.

Results and Conclusion: $61 \%$ of the Acinetobacter species were multidrug resistant of which $63 \%$ had efflux pump as detected by chloramphenicol substrate which is better than Tetracycline $(\mathrm{p}<0.05)$. A difference of $5 \mathrm{~mm}$ in the zone diameter can be used to detect efflux pump. The gene encoding for the efflux pump - AdeFGH was present in $72 \%$ of the isolates. NMP can be a potential adjuvant with common antibiotics to treat MDR Acinetobacter infections.
\end{abstract}

Keywords: Efflux pump, Acinetobacter, Naphthyl-Methyl-Piperazine.

\section{Introduction}

Acinetobacter, a Gram negative bacterium of emerging complications, has proven to be recently important to research due to multidrug resistance. Its resultant infections have been troublesome especially within intensive care units where contact transmission is prevalent. Such contact transmission is facilitated by indwelling devices which cause bacteremia, ventilator associated pneumonia, meningitis, urinary tract infection, central line infections and wound infections. It is responsible for almost $45 \%$ of the ventilator associated pneumonias in tertiary care hospital across the world. ${ }^{1}$ It is responsible for $48 \%$ of mortality due to pneumonia \& sepsis along with another non fermenting bacilli Pseudomonas. ${ }^{2}$ Multidrug resistance of Acinetobacter is directed against antibiotics such as Aminoglycosides, Penicillins, Monobactams, Carbapenems, Cephalosporins, Quinolones and many others. In India, carbapenem resistance ranges from 9.1-100\% $\%^{3,4}$ in Acinetobacter spp. isolated from various patient populations in different sample types, mostly consisting of respiratory specimens and wound swab samples. Mechanisms of antibiotic drug resistance have been studied in detail and include enzyme production, presence of efflux pumps, alteration of antibiotic binding sites and alteration of metabolic pathways. ${ }^{5}$ In regards to Acinetobacter, the presence of efflux pump primarily the Resistance-Nodulation-Cell
Division (RND) type pump is governed by the expression of AdeFGH gene. Efflux pump inhibitors (EPIs) viz Phenylalanine-Arginine $\beta$-Naphthylamide (PABN), 1-(1naphtylmethyl)-piperazine (NMP) and carbonyl cyanidem-chlorophenylhydrazone (CCCP) are compounds which mechanically inhibits the efflux pump in bacteria resulting in increased concentration of antibiotic intracellularly, thereby killing the bacteria. ${ }^{6}$ The efflux pump inhibitor can be used to study the presence or absence of the efflux pump in a particular isolate. 1-(1-naphtylmethyl)piperazine (NMP) has been shown to be effective in case of inhibiting efflux pumps of Acinetobacter species. ${ }^{7}$ Limited amounts of studies have taken place in India regarding the efflux pump as a mechanism for resistance and the EPIs as a possible adjuvant to antibiotics. This study aims to establish the burden of resistance due to efflux pump in Indian scenario. Most of the studies have used the broth dilution method to study the change in Minimum inhibitory concentration (MIC) of antibiotics. ${ }^{8}$ The procedure is labor intensive and time consuming. Other disadvantage include greater rates of contamination and reader bias in interpretation of MIC. To overcome the disadvantages of broth dilution method, disc diffusion method was adopted and the zone diameter cut off was determined. 


\section{Materials and Methods}

This study was a prospective cross sectional study conducted at a tertiary care hospital. Inclusion criteria being Acinetobacter species isolated from all samples which are atleast resistant to 3 groups of antibiotics viz Cephalosporin, Fluoroquinolone, Aminoglycoside. The identification and susceptibility were done using conventional methods using MacConkey's agar, routine biochemical reactions and Kirby Bauer disk diffusion methods. Mueller Hinton agar with the Efflux pump inhibitor - Naphthyl Methyl Piperazine $(100 \mu \mathrm{g} / \mathrm{ml})$ was prepared. These plates were then blinded by labelling the plates with random numbers (generated using SAS software) for 2 groups. One group of numbers were for plain MHA plates and the other group for MHA with NMP. 2 plates (1 from each group) were packed together and used for conducting the experiment.

One set of plates as packed previously was used for each isolate of Acinetobacter species. A suspension of these isolates were prepared using peptone water and matched to $0.5 \mathrm{McFarland}$ standard. Then they were inoculated as a lawn culture on the agar plates and Chloramphenicol \& Tetracycline disks (Efflux Pump substrates) were placed. The zone of inhibition around the disc was measured on both the petri plates and recorded on excel sheet.

The AdeFGH gene coding for efflux pump was detected by conventional Polymerase chain reaction technique. The following primers (forward \& reverse) were used. ${ }^{9}$ The amplified products were detected by gel electrophoresis method.

\section{P1: GGATTATGGCGACAGAAGGA - 104 bp P2: AATACTGCCGCCAATACCAG}

\section{Results and Discussion}

A total of 267 isolates of Acinetobacter were collected from different clinical specimen submitted to the microbiology laboratory. Endotracheal aspiration was the commonest $(55 \%)$ clinical specimen which yielded Acinetobacter. This frequency of isolation is similar to other studies where an average frequency of isolation was $48 \% .^{10}$ This indicates that the Acinetobacter colonizes the endotracheal tube commonly and causes pneumonia in artificially ventilated patients. Isolation from endotracheal aspiration is one of the criteria to diagnose ventilator associated pneumonia (VAP) according to the IDSA guidelines.

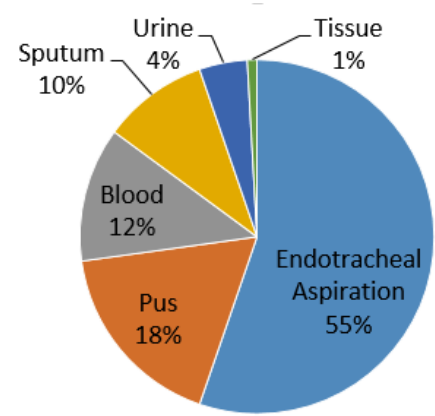

Fig. 1: Clinical Samples that yielded Acinetobacter

These 267 isolates were tested further for presence of efflux pump using Chloramphenicol \& Tetracycline discs. This was done by placing the discs onto a lawn culture of the isolate on MHA plates with and without the NMP. The difference in zone diameter was calculated. Positive for efflux pump was arbitrarily defined as $\geq 5 \mathrm{~mm}$ difference between the zone diameter as observed around discs with and without the NMP.

Table 1: Acinetobacter species positive for efflux pump

\begin{tabular}{|l|c|c|}
\hline Antibiotic Disc & $\begin{array}{c}\text { No. of isolates } \\
\text { showing positive }\end{array}$ & Percentage \\
\hline Chloramphenicol & 169 & 63.2 \\
\hline Tetracycline & 122 & 45.6 \\
\hline
\end{tabular}
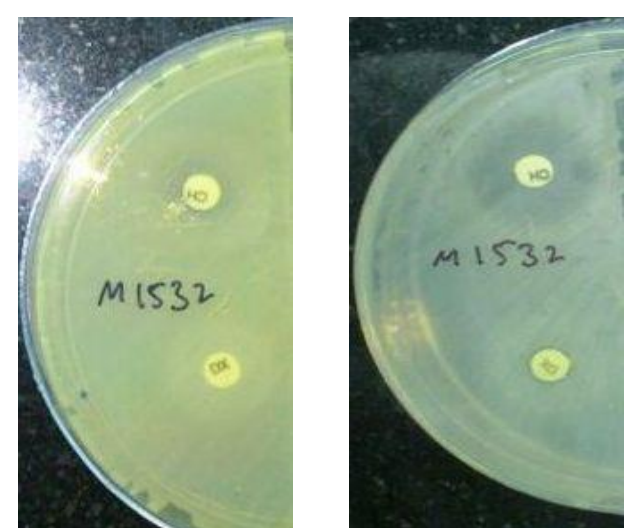

Legend - Left picture is plain MHA showing resistance (no zone of inhibition) \& right picture showing MHA with PABN showing sensitive (large zone of inhibition)

Fig. 2: Mueller Hinton agar plate with Efflux pump substrate disc 
More than half of the isolates showed presence of efflux pumps when tested with Chloramphenicol. The combination of efflux pump inhibitor - NMP and its significant effect on Chloramphenicol has been shown in the study conducted by Stefannie et al. ${ }^{10}$ Hence Chloramphenicol is a better marker $(p<0.005)$ to detect the efflux pump than Tetracycline as seen in this study.

The above results of detecting efflux pumps with Chloramphenicol disc was with the assumption of 5 $\mathrm{mm}$ difference in zone diameter being taken as significant. To check whether this is actually true in detecting the presence of efflux pumps and not as a confounding factor, the analysis of the results obtained were re-run using different cut off values viz $3 \mathrm{~mm}, 4$ $\mathrm{mm}, 5 \mathrm{~mm}, 6 \mathrm{~mm} \& 7 \mathrm{~mm}$. T test was applied to determine the $\mathrm{p}$ value to establish the significance difference between the zone diameters. This was done only Chloramphenicol disc because the results obtained using this disc were better than Tetracycline disc. The following table shows the $\mathrm{p}$ values obtained for each these cut off zone diameters.

Table 2: $p$ value for various cut off value for zone diameter difference

\begin{tabular}{|l|c|l|}
\hline $\begin{array}{c}\text { Difference in } \\
\text { zone diameter }\end{array}$ & p value & Interpretation \\
\hline $3 \mathrm{~mm}$ & 0.742 & Not significant \\
\hline $4 \mathrm{~mm}$ & 0.146 & Not significant \\
\hline $5 \mathrm{~mm}$ & 0.004 & Significant \\
\hline $6 \mathrm{~mm}$ & 0.926 & Not significant \\
\hline $7 \mathrm{~mm}$ & 0.995 & Not significant \\
\hline
\end{tabular}

It can be observed that when the cut off of $5 \mathrm{~mm}$ is considered only then there is a significant difference between the groups which show presence of efflux pump and those which do not. This indicates that the assumption of $5 \mathrm{~mm}$ as cut off was indeed a correct value to detect the presence of efflux pumps. The literature available on the detection of efflux pumps by EPIs have used broth dilution methods to demonstrate 4 fold reduction in MIC of antibiotics. The broth dilution method is cumbersome and labor intensive. The biggest disadvantage being subjective interpretation of the turbidity after incubation. Since there are no studies which used disc diffusion method, it was difficult to decide on the cut off value to consider positivity for efflux pump. This additional analysis using different cut off values was done to overcome this deficiency and establish a cut off value for disc diffusion procedure.

The efflux pump gene - AdeFGH was detected by conventional PCR technique which showed $72 \%$ of the isolates had this gene. Since efflux pump was detected phenotypically in $63.2 \%$ of the isolates it can be concluded that there are many factors involved in expression of the gene. ${ }^{6}$ The expression of these efflux pumps depends on a two-component regulatory system. Level of the expression of the pump, regulated by such a system, is dependent on the presence of the stimulus (substrate) in environment of the cell, which activating the pump for removing substrate outside the cell. Antibiotics can serve as inducers and regulate the expression of some efflux pumps at the level of gene transcription or mRNA translation, by interacting with regulator systems.

\section{Conclusion}

Acinetobacter, commonly isolated from endotracheal secretions from artificially ventilated patients exhibits efllux pump as the major mechanism of drug resistance. It can be detected by Naphthyl Methyl piperazine as the inhibitor. Chloramphenicol is a better substrate as compared to Tetracycline in detecting efflux pumps. Increase in zone size of $5 \mathrm{~mm}$ in presence of EPI indicates the presence of efflux pump.

EPIs like NMP can be a potential adjuvant drug that can be used clinically after modification to reduce its toxicity. Around $60 \%$ of the infections can be cured with a combination of conventionally used antibiotics along with EPI.

\section{Acknowledgments}

The authors thank Rajiv Gandhi University of Health Sciences, Karnataka for funding the project.

Funding: No funding sources.

Conflict of interest: None declared.

\section{References}

1. Quartin AA, Scerpella EG, Puttagunta S, Kett DH. A comparison of microbiology and demographics among patients with healthcare-associated, hospital-acquired, and ventilator-associated pneumonia: a retrospective analysis of 1184 patients from a large, international study. BMC Infect Dis 2013;13:561.

2. Mohanty S, Maurya V, Gaind R, Deb M. Phenotypic characterization and colistin susceptibilities of carbapenem-resistant of Pseudomonas aeruginosa and Acinetobacter spp. J Infect Dev Ctries 2013;7(11):88087.

3. Gaur A, Garg A, Prakash P, Anupurba S, Mohapatra TM. Observations on carbapenem resistance by minimum inhibitory concentration in nosocomial isolates of Acinetobacter species: an experience at a tertiary care hospital in North India. J Health Popul Nutr 2008;26(2):183-88.

4. Uma Karthika R, Srinivasa Rao R, Sahoo S, Shashikala P, Kanungo R, Jayachandran S, et al. Phenotypic and genotypic assays for detecting the prevalence of metallobeta-lactamases in clinical isolates of Acinetobacter baumannii from a South Indian tertiary care hospital. $J$ Med Microbiol 2009;58(Pt 4):430-35.

5. Singh H, Thangaraj P, Chakrabarti A. Acinetobacter baumannii: A Brief Account of Mechanisms of Multidrug Resistance and Current and Future Therapeutic Management. J Clin Diagn Res JCDR 2013;7(11):26025.

6. Mahamoud A, Chevalier J, Alibert-Franco S, Kern WV Pagès J-M. Antibiotic efflux pumps in Gram-negative 
bacteria: the inhibitor response strategy. J Antimicrob Chemother 2007;59(6):1223-29.

7. Coban AY, Guney AK, Tanriverdi Cayci Y, Durupinar B. Effect of 1-(1-Naphtylmethyl)-piperazine, an efflux pump inhibitor, on antimicrobial drug susceptibilities of clinical Acinetobacter baumannii isolates. Curr Microbiol 2011;62(2):508-11.

8. Bina XR, Philippart JA, Bina JE. Effect of the efflux inhibitors 1-(1-naphthylmethyl)-piperazine and phenylarginine-beta-naphthylamide on antimicrobial susceptibility and virulence factor production in Vibrio cholerae. J Antimicrob Chemother 2009;63(1):103-8.

9. Wieczorek P, Sacha P, Hauschild T, Zórawski M, Krawczyk M, Tryniszewska E. Multidrug resistant Acinetobacter baumannii--the role of AdeABC (RND family) efflux pump in resistance to antibiotics. Folia Histochem Cytobiol 2008;46(3):257-67.
10. Pannek S, Higgins PG, Steinke P, Jonas D, Akova M, Bohnert JA, et al. Multidrug efflux inhibition in Acinetobacter baumannii: comparison between 1-(1naphthylmethyl)-piperazine and phenyl-arginine-betanaphthylamide. J Antimicrob Chemother 2006;57(5):970-74.

How to cite this article: T. Sandeep, A. Indumathi, S. Prasanthi. Detection of efflux pump in multidrug resistant acinetobacter Species using Naphthyl-MethylPiperazine. Int J Med Microbiol Trop Dis 2018;4(4):22629. 